\section{L'Actualité économique}

L'ACTUALITÉ

ÉCONOMIQUE

\section{Inventaire des documents relatifs à l'histoire économique du Canada français - 2}

\section{Cameron Nish}

Volume 43, numéro 2, juillet-septembre 1967

URI : https://id.erudit.org/iderudit/1000154ar

DOI : https://doi.org/10.7202/1000154ar

Aller au sommaire du numéro

Éditeur(s)

HEC Montréa

ISSN

0001-771X (imprimé)

1710-3991 (numérique)

Découvrir la revue

Citer ce document

Nish, C. (1967). Inventaire des documents relatifs à l'histoire économique du Canada français - 2. L'Actualité économique, 43(2), 374-411.

https://doi.org/10.7202/1000154ar
Ce document est protégé par la loi sur le droit d'auteur. L'utilisation des services d'Érudit (y compris la reproduction) est assujettie à sa politique d'utilisation que vous pouvez consulter en ligne.

https://apropos.erudit.org/fr/usagers/politique-dutilisation/ 


\title{
Inventaire des documents relatifs à l'histoire économique du Canada français - $2^{*}$
}

\author{
1740 \\ C $11 \mathrm{~A}$
}

1142. Marly, 15 mai 1740. «Estat des payemens que le Roy veut et ordonne estre faits par Me Barthelemy moufle de la Tuillerie Tresorier générale de la marine, pour les Depenses mentionnées cy apres faites et a faire pour le service de sa Majesté au Pays de Canada pendant l'année 1740. 》 (Dépenses générales pour achats de marchandises et autres besoins de la Colonie; appointements des officiers généraux et autres; solde des compagnies; gages et entre tien des employés des magasins et bureaux : Québec, Trois-Rivières, Montréal ; loyers de maisons et bureaux : Québec, Montréal ; gra' tifications ordinaires, extraordinaires: Québec, Montréal, TroisRivières ; autres dépenses ; fortifications et réparations ; récapitulation.) f $311-315$, pp. 274-284.

114-2. Marly, 15 mai 1740. «Estat des Payemens que le Roy veut et ordonne estre faits par Me Barthelemy moufle de la Tuillerie Tresorier général de la Marine pour les Depenses mentionnées cy après faites et a faire pour le service de sa Majesté au pays de $\mathrm{Ca}$ nada pendant l'année 1740 Sur les fonds provenant de la remise qui doit estre faites au Tresorier général par le fermier general du Domaine Doccident du produit des droits du domaine qu'il perceoit

- Pour la première partie de ce document, voir L'Actualité Économique, avril. juin 1967. 
en fance. 》 (Officiers de guerre et garnisons entretenus : Québec, Montréal, Trois-Rivières ; maisons religieuses : Québec, Montréal ; officiers de Justice: Québec, Montréal, Trois-Rivières; hôpital de Québec ; dépenses extraordinaires ; dépenses de l'Isle Royale ; dépenses de la Louisiane; récapitulation.) F 316-319, pp. 285-292.

1142. Québec, 30 août 1740. «Toisé des Ouvrages de Maçonnerie faits par le S Jean Maillon Entrepreneur aux Exhausse,ems des Murs Servant de Toures a l'Isle d'Orleans sur le bord du fleuve St. Laurent pour servir de Balize aux Vaisseaux qui font la Traverse faits pendant l'année mil sept cent quarante. » (Mur à la Côte Saint Jean.) Signé Hocquart, Varin. f 326, pp. 301-302.

1141. Québec, 20 octobre 1742. «Bordereau des Recettes et Dé, penses faites par le Sr Taschereau Commis en ce Pays de Mrs les Trésoriers généraux de la Marine pendant l'année 1740 ainsy qu'il en suit. » (Recettes : fonds ordonnés par l'état du Roy ; à déduire les dépenses faites en 1738 et 1739 ; recette extraordinaires : reçu des Ecclésiastiques de Montréal; imposition sur les bourgeois de Montréal ; produit des congés ; ventes de marchandises, vivres et pelleteries; ventes au navire Le Rubis; ventes des magasins du Roy: Montréal, Trois-Rivières. Dépenses: achats de marchandises; construction et radoub des bateaux et canots: Québec, Trois-Rivières, Montréal, Chambly ; courses et voyages : Québec, Montréal, Trois-Rivières ; fret et voiture : Québec, Trois-Rivières, Montréal ; journées d'ouvriers et façons d'ouvrages : Québec, TroisRivières, Montréal, Chambly, Niagara ; achats de marchandises : munitions de guerre et de bouche : Québec, Trois-Rivières, Montréal ; vivres : Québec, TroisRivières, Montréal ; achats de bois a brûler: Québec, Trois-Rivières, Montréal ; hôpitaux: Québec, Trois-Rivières, Montréal ; dépenses imprévues: Québec, TroisRivières, Montréal, Chambly, Frontenac, St. Fréderic ; appointe ments d'officiers généraux et autres ; appointements des officiers reformés ; solde des compagnies : Québec, Trois-Rivières, Montréal ; officiers détachés; gages et entretien des employés dans les bureaux et magasins : Québec, Trois-Rivières, Montréal, Chambly, Frontenac, Niagara, St. Fréderic ; loyers des maisons : Québec, Montréal ; gratifications ordinaires ${ }^{1}$, extraordinaires : Québec, Montréal,

I. Fin du volume $8 \times 4^{\cdot x}$. 
Trois-Rivières ; autres dépenses ; fortifications : Montréal ; réparations : Québec, Trois:Rivières, Montréal, Chambly, Prontenac, Niagara, St. Fréderic ; artillerie; total de la dépense; déduction de fonds faits en 1741 et 1742.) Signé Hocquart, Varin. f 261-298, pp. 169-250 (vol. 1141) et f 298-306, pp. 251-267 (vol. 114-2).

1142. «Balance pour connoistre de quels Chapitres procedent les Excedents de depense de L'année 1740. » (Chapitre : construction et radoub de bateaux et canots ; courses et voyages; fret et voiture ; journées d'ouvriers et façons d'ouvrages ; achats de marchan dises et munitions ; bois a brûler ; hôpitaux ; dépenses imprévues ; appointements des officiers généraux; officiers reformés ; solde des compagnies; gages et entretien d'employés; loyers de maisons et bureaux ; gratifications ordinaires, extraordinaires; autres dépenses ; fortifications ; réparations ; artillerie.) f 308-310, pp. 268-273.

1141. «Bordereau du Compte a rendre par le Commis du Tréso rier Général de la Marine a Quebec de la Recette et Depense par lui faite pour le Domaine d'Occident l'année mil sept Cent quarante. \ (Total de la recette; payements des charges ordonnés par l'état du Roy ; total de la dépense.) f 208-209, pp. 94-95:

1141. «Bordereau du Compte Rendu par le Commis du Trésorier Général de la Marine a Québec de la Recette et Dépense par lui faite pour le Domaine d'Occident pendant L'année mil sept cens quarante.». (Recettes : produit net ; traite sur le Trésorier Général de la Marine; total de la recette. Dépenses : paye,ents des charges ordonnés par l'état du Roy ; total de la dépense.) f 248, pp. 149. 151.

1141. « Bordereau du Compte Rendu par le Directeur Receveur du Domaine d'occident de la Recette et Dépense par lui faite du produit des Droits du Domaine pendant l'année mil sept cent quarante. 》 (Recettes : droits d'entrées : sur l'eau de vie, sur les guidives; droits de sorties : en argent, en nature ; lods et ventes; bail de Tadoussac ; total. Dépenses : pour les magasins du Roy à Québec ; pour achats de rafraîchissements ; pour les malades du vaisseau Le Rubis ; frais extraordinaires de Justice ; nourritures et entretien d'enfants bâtards ; papier terrier ; frais de régie ; total.) f 249, pp. $152-153$. 
1141. Québec, 29 octobre 1741. «Hocquart au Ministre. » (Commentaire sur le compte du Domaine d'Occident pour l'année 1740.) Signé Hocquart, f 255-256, pp. 160-161.

Voir aussi : 1739. 1141. Québec, 20 octobre 1741. «Bordereau... 1739. 》f 98-134, pp. 149-201 et 1-11.

Voir aussi : 1742. 1141. Québec, 16 août 1742. «Bordereau... Cugnet... 》f 231-232, pp. 129-132.

Voir aussi : 1743. 1141. Québec, 28 octobre 1743. «Hocquart au Ministre. »f 237-240, pp. 138-140.

Voir aussi : 1743. 115-1. Québec, 15 octobre 1748. « Memoire... 》 t 62, pp. 144145.

Voir aussi : 1748. 116-1. Québec, 1 novembre 1748. «Borde reau... acquit... »f 122, p. 214.

\section{1 \\ C $11 \mathrm{~A}$}

1142. Québec, 31 octobre $1740^{2}$. «Memoire sur le Projet des Depenses a faire en Canada pendant L'Année 1741 comparaison aux precedents Etats du Roy. 》 Signé Hocquart. f 320-325, pp. 293-300.

114-2. «Projet de l'Etat des payements que le Roy veut et ordonne être faits par Me Florent Deselle $\&$ etc. » (Pour achats de mar. chandises et autres besoins de la Colonie; appointements des offciers généraux et autres; appointements des officiers reformés; solde des compagnies; gages et entretien des employés : Québec, TroisRivières, Montréal, Chambly, Frontenac, Niagara, St. Fréderic ; loyers de maisons et bureaux : Québec, Montréal ; gratifications or' dinaires, extraordinaires : Québec, Montréal, Trois-Rivières ; autres dépenses; fortifications; réparations; récapitulation.) f 327.335 , pp. 307-315.

115-2. Marly, 14 mai 1741. «Estat des Payemens que le Roy veut et ordonne estre faits par Mr Marcelin françois Zacharie de Selle Tresorier Général de la marine, pour les Depenses mentionnées cy apres faites et a faire pour le service de Sa Majesté au pays de $\mathrm{Ca}$.

2. Ce document porte aussi la date suivante: Versailles, 16 février, 1741 . 
nada pendant l'année 1741. 》 (Construction et radoub de canots et bateaux ; courses et voyages ; fret et voiture ; journées d'ouvriers et façons d'ouvrages; achats de marchandises et munitions; bois à brûler ; présents a faires aux Sauvages ; subsistance et remèdes pour les soldats malades; dépenses imprévues; appointements des off. ciers généraux et autres; appointements des officiers reformés; solde des compagnies; gages et entretiens des employés dans les magasins et bureaux; loyers de maisons et bureau : Québec, Montréal ; gratifications ordinaires, extraordinaires: Québec, Montréal, TroisRivières; autres diverses dépenses; fortifications; réparations; récapitulation.) f 5.9, pp. 8-22.

115-1. Marly, 14 mai 1741. «Pour les Depenses de Canada a payer du fonds du Domaine D'occident de 1741.» (Officiers de guerre et garnisons entretenus : Québec, Montréal, Trois-Rivières ; maisons religieuses : Québec, Montréal ; officiers de Justice : Québec, Montréal ; autres officiers de Justice ; hôpital de Québec ; dépenses extraordinaires pour les frais de Justice; dépenses de l'Isle Royale; dépenses de la Louisiane; récapitulation.) f 10-13, pp. 23-33.

117-1. Michilimackinac, 12 juillet 1741. «Memoire du Bléd que moy La Croix ay fourny a Monsieur de Blainville, pour le Service du Roy. » (Fournitures.) Signé Hubert et Compagnie, Blainville, Beauharnois, Hocquart, Varin, f 347, p. 210.

1141. Québec, 26 octobre 1741. «Etat des Liqueurs et vins de Liqueurs debarqués des Batiments En la Rade de Quebec Année 1741. » Signé Dusautoy, f 250-252, pp. 154-157.

1141. Québec, 26. octobre 1741. «Etat des Tabacs Tant en Carottes de Saint Domingue qu'en Carottes de Saint Vincent Et poudre d'Espagne debarqués des bâtiments arrivés en la rade de Québec en l'année 1741. 》 Signé Dusautoy. f 253-254, pp. 158-159.

1142. Québec, 20 octobre 1742. «Bordereau des Recettes et dépenses Faites par le Sr Taschereau Commis en ce Pays de Mrs Les Tresoriers généraux de la Marine pendant L'année 1741. Ainsi qu'il ensuit. » (Recettes : fonds ordonnés par l'état du Roy : déduires les fonds pour les dépenses de 1739 et 1740 ; recettes extraordinaires : reçu des Ecclésiastiques de Montréal ; impositions sur les bourgeois 
de Montréal ; produit de 25 congés; ventes des magasins : Québec, Trois-Rivières, Montréal. Dépenses : Construction, radoub et entretien des bateaux et canots: Québec, Trois-Rivières, Montréal ; courses et voyages.: Québec, Montréal ; fret et voitures : Québec, Montréal, Trois-Rivières ; journées d'ouvriers et façons d'ouvrages : Québec, Trois-Rivières, Montréal ; achats de marchandises et munitions de guerre et de bouche: Québec; marchandises et munitions : Trois-Rivières, Montréal ; vivres : Québec, Trois-Rivières, Montréal ; achats de bois a brûler : Québec, Trois-Rivières, Montréal ; hôpitaux : Québec, Trois-Rivières, Montréal ; dépenses imprévues: Québec, Trois-Rivières, Montréal, Chambly, Frontenac, Niagara, St. Fréderic ; appointements des officiers généraux et autres; appointements des officiers reformés; solde des compagnies : Québec, Trois-Rivières, Montréal ; officiers détachés; gages et entretien des employés dans les magasins et bureaux : Québec, TroisRivières, Montréal, Chambly, Frontenac, Niagara, St. Fréderic ; loyers de maisons et bureaux : Québec, Montréal ; gratifications ordinaires, extraordinaires : Québec, Trois-Rivières, Montréal ; autres diverses dépenses ; fortifications ; réparations : Montréal, Québec, Trois-Rivières, Chambly, Frontenac, Niagara, St. Fréderic ; artillerie. A déduire les fonds faits pour 1742 et les'fonds demandés pour 1743.) Signé Hocquart, Varin. f 336-402, pp. 316-437.

115 1. «Balance pour Connoistre dequels Chapitres procedent les Excedents de depense de L'année 1741.» (Chapitre : construction et radoub des canots ; courses et voyages ; fret et voiture ; journées. d'ouvriers et façons d'ouvrages; achats de marchandises et munitions ; bois à brûler ; hôpitaux ; dépenses imprévues ; appointements des officiers généraux et autres; officiers reformés ; solde des compagnies; gages et entretien d'employés ; loyers de maisons et bureaux ; gratifications ordinaires, extraordinaires ; autres diverses dé penses ; fortifications ; réparations ; artillerie.) f 2-5, pp. $2-7$.

$1141 .{ }^{3}$ «Bordereau du Compte a rendre par le Directeur Receveur Domaine d'occident de la Recette et Dépense par lui faite du pro duit des Droits du Domaine pendant l'année mil sept cent quarante un. » (Recettes : droits d'entrée ; droits de sorties; droits seigneu-

3. Ce document porte aussi le titre de « Domaine d'occident, Canada, Année 1742. Le document qui suit, $f 245 \cdot 246$, pp. $147 \cdot 148$, est aussi un bordereau pour l'année $\mathrm{r} 74 \mathrm{r}$. 
riaux ; bail de la ferme de Tadoussac ; total de la recette. Dépenses : d'entrées ; pour le compte du Roy ; remise de droits ; frais de Jus tice ; papier terrier ; appointements ; total de la dépense.) f 243-244, pp. 145-146.

1141. «Bordereau du Compte a rendre par le Commis du Treso rier Général de la Marine a Québec de la Recette et Depense par lui faite par le Domaine d'occident pendant l'année mil sept cent quarante un. » (Recettes : produit net ; traite sur le Trésorier Général ; total de la recette. Depenses : payements des charges ordonnés par l'état du Roy.) f 245-246, pp. 147-148.

1141. « Bordereau du Compte a rendre par le Directeur Receveur du Domaine d'Occident de la Recette et Dépense par lui faite du Produit des Droits du Domaine pendant l'année mil sept cent quarante un. 》 (Recettes : droits d'entrées : sur les vins et guildives ; droits de sorties ; droits seigneuriaux ; bail de la ferme de Tadous $\mathrm{sac}$; total de la recette. Dépenses : pour le compte du Roy : remises de droits ; frais de Justice ; papier terrier; appointements et frais du régie ; total de la dépense.) f 257, pp. 162-163.

114.1. « Bordereau du Compte a Rendre par le Commis du Trésorier General de la Marine a Quebec de la Recette et Dépense par lui faite pour le Domaine d'Occident pendant l'année mil sept cens quarante un. » (Recettes : produit net; traite sur le Trésorier général de la Marine; total de la recette. Dépenses : payements ordonnés par l'état du Roy). f 258, p. 164.

1141. «Bordereau du Compte Rendu par le Directeur Receveur du Domaine d'Occident en Canada de la Recette et Depense par lui faite du Produit des Droits du Domaine pendant l'année mil sept cent quarante un. » (Recettes : droits d'entrées; droits de sorties; droits seigneuriaux; bail de Tadoussac ; total de la recette. Dépenses : magasins de sa Majesté; pour les malades; remises; frais extraordinaires de Justice ; papier terrier ; appointements et frais de régie ;'total de la dépense.) f 259, pp. 165-166.

1141. «Bordereau du Compte rendu par le Commis du Tresorier Général de la Marine a Quebec de la Recette et Depense par lui faite de Droits du Domaine d'occident en Canada pendant l'année mil sept cent quarante Un. » (Recettes : produit net; traite sur le 
Trésorier Général. Dépenses : charges ordonnés par l'état du Roy; total de la dépense.) f 260, pp. 167-168.

Voir aussi : 1741. 1141. Québec, 20 octobre 1741. « Bordereau... $1741 \ldots \gg$ f 336, p. 316.

Voir aussi : 1742. 1141. «Québec, 16 août 1742. «Bordereau... Cugnet... »f 231-232, pp. 129-132.

Voir aussi : 1740. 114-2. Québec, le 20 octobre 1742. «Bordereau... 1740. 》f 306, p. 267.

Voir aussi : 1743. 1141. Québec, le 28 octobre 1743. « Hocquart au Ministre. 》f 237-240, pp. 138-140.

Voir aussi : 1743. 115-1. Québec, le 15 octobre 1748. «Memoire... 》 f $62 \cdot 63$, pp. 144146 .

\section{2 \\ C $11 \mathrm{~A}$}

115-1. « Projet de l'etat des payements que le Roy veut et ordonne être faits par Me Barthelemy Moufle de la Tuillerie... » (Construc tion et radoub des bateaux et canots; courses et voyages; fret et voiture; journées d'ouvriers; bois à brûler; présents à faire aux Sauvages ; subsistance et remède ; appointements des officiers généraux et autres; appointements des officiers reformés; solde des compagnies; gages et entretien des employés dans les magasins et bureaux ; loyers de maisons et bureaux : Québec, Montréal ; gratifications ordinaires, extraordinaires: Québec, Montréal, Trois Rivières; autres dépenses ; fortifications ; réparations ; récapitulation.) f 14-20, pp. 35-48.

115-1. Fontainebleau, 22(2) avril 1742. «Estat des Payements que le Roy veut et ordonne estre faits par Me Barthelemy moufle de la Tuillerie Tresorier général de la marine pour les Depenses mentionnées cy après faites et a faire pour le Service de Sa Majesté au Pays de Canada pendant l'année 1742. \(Construction de bateaux et radoub de canots ; courses et voyages ; fret et voiture ; journées d'ouvriers et façons d'ouvrages; achats de munitions et marchandises; présents a faire aux Sauvages; bois a brûler ; subsistance et remèdes ; dépenses imprévues ; appointements des officiers généraux et autres; appointements des officiers reformés ; solde des compagnies; gages et entretiens des employés dans les bureaux et maga- 
sins : Québec, Montréal ; loyers de maisons et bureaux : Québec, Montréal ; gratifications ordinaires, extraordinaires : Québec, Montréal ; autres diverses dépenses ; fortifications ; réparations ; récapitulation.) f 21-26, pp. 49-65.

115-1. Marly, 22 avril 1742. «Pour les Depenses de Canada a payer du fonds du Domaine d'Occident de 1742. » (Officiers de guerre et garnisons entretenus : Québec, Montréal, Trois-Rivières ; maisons religieuses : Québec, Montréal ; officiers de Justice : Québec, Montréal, Trois-Rivières; hôpital de Québec; dépenses de l'Isle Royale; dépenses de la Louisiane; récapitulation.) f 27.31, pp. 66-77.

117-1. Michilimackinac, 20 mai 1742. «Memoire de Bléd que moy Le Croix fils ai fourny a Monsieur de Celeron, pour le service du Roy. » (Fournitures.) Signé H. Hubert et Compagnie, Céleron, Beauharnois, Hocquart, Varin. f 346, p. 209.

1141. «Vente des Effets provenans des Traittes du Domaine d'Occident pendant l'année mil sept cent Quarante deux. » (Effets reçus à Québec ; ventes des effets.) f 218-219, pp. 117-120.

1141. Québec, 16 août 1742. «Bordereau de la Recette et dépen ses faites par M Cugnet Directeur et Receveur du Domaine d'Occident pendant les années 1739, 1740, 1741 et 1742 tant des droits du dit Domaine que des frais Extraordinaires de Justice et de la Regie ainsi qu'il en suit. 》 (Recettes : 1739, 1740, 1741, 1742; total de la recette. Dépenses : 1739, 1740, 1741, 1742. Reprises.) Signé Hocquart, Varin, Cugnet. f 231-232, pp. 129-132.

1141. Québec, 29 octobre 1742. «Extrait de la Recette et produit des droits du Domaine D'Occident en Canada pendant la présente année 1742. 》 (Droits d'entrées. Droits de sorties. Droits seigneuriaux. Bail de la ferme de Tadoussac.) Signé Nouchet. f 233, p. 133.

1141. «Bordereau du Compte a rendre par le Receveur du Do maine d'Occident de la Recette et Depense par lui faite du produit des Droits du Domaine pendant l'année mil sept cent Quarante deux. 》(Recettes : droits d'entrées; droits de sorties; droits seigneuriaux; ferme de Tadoussac; total de la recette. Dépenses: frais extraordinaires de Justice ; nourriture d'enfants bâtards ; pa- 
pier terrier ; frais de régie; total de la dépense.) f 241, pp. 141 . 142.

Voir aussi : 1741. 1142. Québec, 20 octobre 1742. «Bordereau... $1741 \ldots \gg$ f 402 , p. 436.

Voir aussi : 1740. 1142. Québec, 20 octobre 1742. « Bordereau... $1740 \ldots \gg$ f 306, p. 267.

Voir aussi : 1743. 1141. Québec, 28 octobre 1743. «Hocquart au Ministre. »f 237-240, pp. 138-140.

Voir aussi : 1744. 117-1. Michilimackinac, 10 juillet 1744. «Mémoire... 》 f 334, p. 196.

Voir aussi : 1745. 117-1. Montréal, 18 juillet 1745. «Memojre... 》f 325 , p. 182.

Voir aussi : 1744. 115-1. Québec, 25 septembre 1746. «Bordereau... $1744 \ldots \gg$ f 65, p. 149.

\section{3 \\ C $11 \mathrm{~A}$}

115-1. Versailles, 9 juin 1743. «Pour les Depenses de Canada pendant l'année 1743. \ (Construction de bateaux et radoub de canots ; courses et voyages; fret et voiture ; journées d'ouvriers et façons d'ouvrages ; achats de munitions et marchandises ; présents a faire aux Sauvages ; bois a brûler ; subsistance et remèdes; dépenses imprévues ; appointements des officiers généraux et autres ; appointements des officiers reformés ; solde des compagnies ; gages et entretien des employés dans les magasins et bureaux : Québec, Trois Rivières, Montréal ; loyers de magasins et bureaux : Québec, Montréal ; gratifications ordinaires, extraordinaires : Québec, Montréal, Trois-Rivières; autres diverses dépenses; fortifications; réparations ; récapitulation.) f 49-55, pp. 111-127.

115-1. Versailles, 9 juin 1743. «Pour les Depenses de Canada a payer du fonds du Domaine d'occident de l'année 1743. » (Offciers de guerre et garnisons entretenus : Québec, Montréal, Trois Rivières ; maisons religieuses : Québec, Montréal ; officiers de Justice et de Police : Québec, Montréal, Trois-Rivières; dépenses ex 
traordinaires; dépenses de l'Isle Royale; dépenses de la Louisiane ; récapitulation.) f 56-59, pp. 128-129.

1141. Québec, 23 octobre 1743. «Extrait du Montant des, Droits du Domaine D'occident en Canada pendant la présente année 1743 » (Sur le vin, les liqueurs; eau de vie, l'orignaux; bail de Tadous sac ; droits seigneuriaux). Signé Nouchet. f 247, p. 149.

1141. Québec, 25 octobre 1743. «Etat des Tabacs Tant en Ca' rottes de St Domingue qu'en Carottes de St. Vincent Et poudre d'Espagne debarqués des Batiments arrivés En la Rade de Quebec En l'année 1743. » Signé Dusautoy. f 242, p. 143.

1141. Québec, 28 octobre 1743. «Hocquart au Ministre. ». (Don' ne le compte de régie du Directeur du Domaine d'Occident pour les années $1740,1741,1742$ et 1743). Signé Hocquart. f 237-240, pp. 138-140.

115-1. Québec, 25 octobre 1744. «Etat de la Recette du produit des Postes des Pays d'en Haut pour l'année 1743 et des Payements faits sur le produit de ces postes par M. le $\mathrm{M}^{\prime}$ is de Beauharnois en conformité des Ordres de Sa Majesté. 》(Recettes des congés ; paye' ments ; récapitulation.) Signé Beauharnois. f 33, pp. 78-79.

115-1. Québec, 15 octobre 1748. «Mémoire au Sujet de la Situation des finances du Canada de L'Année mil sept cent quarante trois. 》. (Fonds ordonnés par l'état du Roy: construction de bateaux et radoub de canots ; courses et voyages ; journées d'ouvriers et façons d'ouvrages; achats de marchandises; présents a faire aux Sauvages ; subsistance et remèdes; dépenses imprévues ; appointe ments des officiers généraux et autres; appointements des officiers reformés ; solde des compagnies; gages et entretien des employés ; loyers de maisons et magasins ; gratifications ordinaires, extraordinaires ; fortifications. Fonds de l'année 1744, 1745. Recettes extraordinaires. Récapitulation. Dépenses : construction, radoub de canots et bateaux ; courses et voyages ; fret et voiture ; journées d'ouvriers; achats de marchandises; hôpitaux; dépensès imprévues; appointements des officiers généraux et autres; appointements des officiers reformés ; solde des compagnies, gages et entretien des em. ployés ; loyers de maisons et bureaux'; gratifications ordinaires, extraordinaires; autres diverses dépenses ; fortifications'; réparations ; 
total de la dépense. Acquits des dépenses rejettés sur 1743 : dépenses faites en 1741, 1740 et 1737. Récapitulation.) Signé Bigot. f 60 . 64 , pp. $140-148$.

115-1. Québec, 25 octobre 1750. «Bordereau des Recette et Depense des acquits Employés dans le Compte de l'Exercice mil sept cent quarante trois. » Recettes : fonds ordonnés par l'état du Roy; reçu du Séminaire de Saint-Sulpice ; reçu de l'imposition de Montréal; produit de la vente des congés; ventes des magasins : Québec, Trois-Rivières, Montréal. Dépenses : construction et radoub de bateaux et canots : Québec, Trois-Rivières, Montréal ; courses et voyages : Québec ; fret et voiture : Québec, Trois-Rivières, Montréal ; journées d'ouvriers et façons d'ouvrages : Québec, Trois-Rivières, Montréal ; achats de marchandises et munitions : en France, à Québec, Trois-Rivières, Montréal ; achats de vivres: Québec, Trois-Rivières, Montréal ; achats de bois a brûler : Québec, TroisRivières, Montréal ; hôpitaux : Québec, Trois-Rivières, Montréal ; dépenses imprévues: Québec, Trois-Rivières, Montréal, Chambly, St. Fréderic ; appointements des officiers généraux et autres ; ap pointements des officiers reformés; solde des troupes: Québec, Trois-Rivières, Montréal ; appointements et gages des employés dans les magasins et bureaux : Québec, Trois-Rivières, Montréal, Frontenac, Niagara, St. Fréderic; loyers de maisons : Québec, Montréal ; gratifications ordinaires, extraordinaires; diverses dépenses : mouvements des pays d'en haut, achat de maisons, réparations: Québec, Trois-Rivières, Montréal, Chambly ; artillerie.) Signé Imbert, Bigot. f 34-48, pp. 80-110.

Voir aussi : 1741. 1142. Québec, 20 octobre 1742. «Bordereau... $1741 \ldots \gg$ f 402, p. 437.

Voir aussi : 1744. 117-1. Michilimackinac, 10 juillet 1744. «Mémoire... » f 334, p. 196.

Voir aussi : 1745. 117-1. Montréal, 18 juillet 1745. «Mémoire... 》f 325, p. 182.

Voir aussi : 1744. 117-1. Détroit, 7 août 1744. «Memoire...» f 344, p. 212.

Voir aussi : 1744. 115- Québec, 25 septembre 1746. «Borde reau... $1744 \ldots \gg$ f 65 , p. 149. 
Voir aussi : 1745. 115-1. Québec, 18 septembre 1747. «Bordereau... 1745... 》f 150 , p. 397.

Voir aussi : 1744. 116-1. Québec, 30 octobre 1748. «Memoire... MVIII' quarante quatre... » f 82, p. 129.

Voir aussi : 1748. 116-1. Québec, 1 novembre 1748. «Borde' reau.. acquits... MVII' Quarante huit. »f 122, p. 215.

Voir aussi : 1749. 116-1. Québec, 3 novembre 1748. «Extraits des fonds ordonner... » f 141, p. 255.

Voir aussi : 1745. 115-2. Québec, 25 octobre 1750. «Bordereau des acquits... 1745. »f 249, p. 207.

\section{4 \\ C $11 \mathrm{~A}$}

115-1. Versailles, 26 avril 1744. «Pour les Dépenses de Canada a payer du fonds du Domaine d'Occident de l'année 1744. » (Offl. ciers de guerre et garnisons entretenus : Québec, Montréal, TroisRivières ; maisons religieuses : Québec, Montréal ; officiers de Justice et de Police: Québec, Montréal, Trois-Rivières; hôpital de Québec; dépenses extraordinaires; dépenses de l'Isle Royale; dé, penses de la Louisiane ; récapitulation.) f 135-138, pp. 358-369.

115-1. Versailles, 26 avril 1744. «Estat des Paymens que le Roy veut et ordonne estre faits par Me Louis Barthelemy Moufle de Georvile, tresorier general de la Marine pour les Dépenses mention. nées cy après faites et a faire pour le Service de Sa Majesté ay pays de Canada pendant l'année 1744. » (Construction des bateaux et radoub de canots; courses et voyages; fret et voiture; journées d'ouvriers et façons d'ouvrages; achats de marchandises; présents a faire aux Sauvages ; bois a brûler ; subsistance et remèdes ; dépen. ses imprévues; appointements des officiers généraux et autres ; ap pointements des officiers reformés; solde des compagnies, gages et entretien des employés dans les magasins et bureaux : Québec, TroisRivières, Montréal ; loyers des magasins et bureaux : Québec, Montréal ; gratifications ordinaires, extraordinaires: Québec, Montréal, Trois-Rivières; autres diverses dépenses; fortifications ; récapitulation.) f $139-145$, pp. $369-384$. 
117-1. Michilimackinac, 10 juillet 1744. «Mémoire des fournitures que moy Charles Douaire, voyageur et Négociant a Missilimakinac ay fourny pour le service du Roy, et par ordre de Monsieur de Verchere, y Commandant pendant les années 1742, 1743 et 1744. 》 (Diverses fournitures, 1742, 1743, 1744.) Signé Douaire, Verchères, Hocquart, Varin. f 334, p. 196.

117-2. Détroit, 1 août 1744. «Memoire des fournitures faites par moy Gouin pour le Roy par l'ordre de Monsieur Le Commandant. » (Fournitures.) Signé Gouin, Navarre, Beauharnois, Hocquart, Va. rin. f 358, p. 221.

117-2. Détroit, 1 août 1744. «Estat des fournitures faites par moy Gouin pour Le Roy par L'ordre de Monsieur le Commandant. » (Fournitures.) Signé Gouin, Longueuil, Navarre, Beauharnois, Hoc' quart, Varin. f 382, p. 247.

117-1. Détroit, 7 août 1744. «Certificat de fournitures pour le Sr. St. Côme. » (Diverses fournitures.) Signé Longueuil, Navarre, Beauharnois, Hocquart, Varin. f 348, p. 211.

117-1. Détroit, 7 août 1744. «Memoire des fournitures faites par moy Jean B'te Bondy pour le compte et service du Roy par ordre de Monsieur de Longueuil, le 24 Septembre 1743. » (Diverses fournitures.) Signé J.B. Bondy, Longueuil, Navarre, Beauharnois, Hoc' quart, Varin. f 344, p. 212.

117-2. Détroit, 7 août 1744. «Memoire des fournitures que j'ay faites pour le service du Roy par ordre de Mr de Longueuil au De' troit, scavoir, moy Jacques La Selle. » (Fournitures.) Signé Jacques La Selle, Longueuil, Navarre, Beauharnois, Hocquart, Varin. f 356, p. 219.

117-2. Détroit, 7 août 1744. «Memoire des fournitures que j’ay faites moy Claude Champagne, pour le Compte et service du Roy, par ordre de Monsieur de Longueuil au Detroit. » (Fournitures.) Signé Longueuil, Navarre, Beauharnois, Hocquart, Varin. f 359, p. 222.

117-2. Détroit, 7 août 1744. «Certificat de fourniture pour le Sieur Sté Côme d'un canot. » (Fournitures.) Signé Longueuil, Navarre, Beauharnois, Varin. f 377, p. 242. 
117-2. Détroit, 7 août 1744. « Certificat de fournitures faites par Claude Champagne.» (Fournitures.) Signé Longueuil, Navarre, Beauharnois, Varin. f 378, p. 243.

117-2. Montréal, 2 septembre 1744. «Certificat de remises de deux femmes Esclaves par Mr De la Verendrye. » (Certificat.) Signé Marin, Beauharnois, Hocquart, Varin. f 357, p. 220.

117-2. Rivière St. Jean, 2 novembre 1744. «Estat de ce qui a été delivré par Michel Bergeron et Joseph Belfontaine dit Beausejour aux sauvages de Koubak pour se rendre au port Royal par ordre de Monsieur Duquesnel. » (Etat.) Signé Germain, Varin. f 379, p. 244.

117-2. Québec, 26 novembre 1744. «Estat des fournitures que jay fait faire L'Eté dernier par ordre de Monsieur le Gouverneur général pour faire festin aux sauvages de la mission de Koubak a L'occasion de la guerre. » (Etat.) Signé Messaiger, Varin. f 380, p. 245.

117-2. Québec, 26 novembre 1744. « Estat des vivres et munitions que jay fait fournir L'Eté dernier, par ordre de Monsieur le Gouverneur général aux sauvages de la mission de Koubak, allant au Port Royal, a l'occasion de la guerre. » (Etat.) Signé Messaiger; Varin. f 381, p. 246.

115-1. Québec, 25 septembre 1746. «Bordereau des Recettes et Dépenses faites par le Sr Taschereau Commis en ce Pays de Mrs Les Trésoriers généraux de la Marine pendant L'année 1744 ainsi qu'il Ensuit. » (Recettes : fonds ordonnés par l'état du Roy ; déduires les dépenses de 1742 et 1743 ; recettes extraordinaires : pro duit des congés ; produits des Postes des Pays d'en haut; ventes des magasins : Québec, Trois-Rivières, Montréal; prix de la vente de la ferme de Frontenac et Niagara. Dépenses : construction et radoub des bateaux et canots : Québec, Montréal ; couses et voyages : Qué bec, Montréal ; fret et voiture : Québec, Montréal ; journées d'ouvriers et façons d'ouvrages: Québec, Trois-Rivières, Montréal ; achats de marchandises et munitions : marchandises et munitions : Québec, Trois-Rivières, Montréal, Niagara, Frontenac, (a déduire lés marchandises livrés au Sr. Chalet ${ }^{4}$ ) ; vivres : Québec, Trois

4. Détenteur du bail de traite des Postes' de Prontenac et Niagara. 
Rivières, Montréal, Niagara ; bois a brûler : Québec, Trois-Rivières, Montréal ; hôpitaux : Québec, Trois-Rivières, Montréal ; dépenses imprévues : Québec, Trois-Rivières, Montréal, Chambly, St. Fré' deric, Frontenac, Niagara ; appointements des officiers généraux et autres ; appointements des officiers reformés ; solde des compagnies ; gages et entretien des employés dans les magasins et bureaux : Québec, Trois-Rivières, Montréal, Frontenac, Niagara, St. Fréderic ; loyers de maisons et bureaux : Québec, Montréal ; gratifications ordinaires, extraordinaires : Québec, Montréal, Trois-Rivières; autres diverses dépenses; fortifications: Québec, Montréal ; artillerie : Québec, Montréal, Sté Fréderic ; réparations : Québec, Trois-Rivières, Montréal, Chambly, Frontenac, Niagara, St. Fréderic ; artillerie : Québec ; total de la dépense. A déduire les sommes cy après : pour les fonds de l'année 1745, 1746 et 1747.) Signé Hocquart, Varin. f 65-118, pp. 149-237.

117-1. Québec, 2 octobre 1746. «Reparations aux fortifications et Batimens Civils 1744 Sur L'Exercice 1747. » (Fonds a remettre pour le payment: Québec, Trois-Rivières, Montréal, Chambly, Frontenac, Niagara ; fonds faits ; reste a remettre.) Signé Hocquart, Varin. f 90, p. 162.

116-1. Québec, 30 octobre 1748. «Memoire au sujet de la Situation des finances du Canada de l'année MVIIc quarante quatre. 》 (L'état du Roy : construction et radoub des bateaux et canots; courses et voyages ; fret et voiture ; journées d'ouvriers; achats de marchandises; présents a faire aux Sauvages; bois a brûler; sub sistance et remèdes ; dépenses imprévues; appointements des offciers généraux et autres; appointements des officiers reformés; solde des compagnies; gages des employés; loyers de maisons et bureaux ; gratifications ordinaires, extraordinaires; autres diverses dépenses ; fortifications ; réparations ; déduction des fonds faits par l'état du Roy de 1743 ; total des fonds ordonnés ; autres fonds or. donnés 1745, 1746, 1747 ; recettes extraordinaires; récapitulation. Dépenses : du bordereau du 25 septembre 1746 ; acquits des dépenses rejetées sur 1744 ; ceux de 1742,1743 , et 1746 ; récapilulation. Balance.) Signé Bigot. f 82-86, pp. 128-136.

115-1. Québec, 25 octobre 1750. «Bordereau des Recettes et Depenses Employés dans le Compte rendu pour l'Exercice 1744. » $\left(\mathrm{Re}^{\text {- }}\right.$ 
cettes : fonds ordonnés par l'état du Roy ; reçu pour les congés ; prix des fermes des Postes du pays d'en haut; prix des fermes des postes du Fort Frontenac et Niagara ; ventes des magasins du Roy : Québec, Trois-Rivières, Montréal. Dépenses : Construction et radoub des bateaux et canots: Québec, Trois-Rivières, Montréal ; courses et voyages : Québec, Montréal ; fret et voiture: Québec, Trois-Rivières, Montréal ; journées d'ouvriers et façons d'ouvrages : Québec, Trois-Rivières, Montréal ; achats des marchandises : mar' chandises et munitions : Québec, Trois-Rivières, Montréal, Frontenac ; achats de vivres : Québec, Trois-Rivières, Montréal ; bois a brûler: Québec, Trois-Rivières, Montréal ; hôpitaux : Québec, Trois-Rivières, Montréal ; dépenses imprévues: Québec, Sté Fréderic ; appointments des officiers généraux et autres ; appointements des officiers reformés; appointement et solde des troupes; Québec, Trois-Rivières, Montréal ; gages et entretiens des employés dans les magasins et bureaux : Québec, Trois-Rivières, Montréal, Fron' tenac, Niagara, Sté Fréderic ; loyers de maisons : Québec, Montréal ; gratifications ordinaires, extraordinaires ; autres diverses dépenses ; mouvements des pays d'en haut ; fortifications ; réparations ; maison Episcopale ; réparations aux bâtiments civils ; artillerie : Québec, Montréal.) Signé Bigot, Imbert. f 119.134, pp. 238, 357.

Voir aussi : 1745. 115 1. Québec, 18 septembre 1747. «Borde reau... $1745 \ldots$... f 150 , p. 397.

Voir aussi : 1743. 115-1. Québec, 15 octobre 1748. «Memoire... »f 61-63, pp. 141-144-146.

Voir aussi : 1746. 116-1. Québec, 31 octobre 1748. « Memoire... $\mathrm{MVII}^{\mathrm{c}}$ quarante six. 》f 88-90, pp. 138-143.

Voir aussi : 1748. 116-1. Québec, 1 novembre 1748. «... par Suplement... »f 81, p. 127.

Voir aussi : 1748. 116-1. Québec, 1 novembre 1748. « Bordereau... acquits... MVIIc Quarante huit. 》f 122, p. 215.

Voir aussi : 1747. 115-2. Québec, 3 novembre 1748. «Memoire... »f 365 , p. 412 . 
Voir aussi : 1745. 115-2. Québec, 25 octobre 1750. «Bordereau des acquits... 1745. 》 f 249, p. 208.

Voir aussi : 1746. 115-2. Québec, 24 octobre 1751. «Bordereau général... 1746. 》f 324-325, p. 338.

Voir aussi : 1746. 115-2. Québec, 15 août 1752. «Bordereau... $1746 \ldots$ » f 278 , p. 256.

Voir aussi : 1746. 115-2. Québec, 15 août 1752. «Bordereau... Additions... 1746. 》f 359-362, pp. 401-406.

\section{5 \\ C $11 \mathrm{~A}$}

117-2. St. Joseph. «Memoire des fournitures faites par Mr Marin de la Perriere et Compagnie pour le service du Roy par lordre de $\mathrm{Mr}$ de Villiers Commandant au poste de St. Joseph. » (Diverses fournitures.) Signé La Perrière Marin et Compagnie, Beauharnois, Hocquart, Varin. f 457, p. 332.

117-1. A la Baye, 4 juin 1745. «Memoire des fournitures de vivres fait par nous dermiers de la Baye aux Chefs Sakis et Renard allant en parole a Montréal par ordre de $\mathrm{Mr}$ de Lusignan Commandant pour le Roy aud Poste. 》(Diverses fournitures.) Signé Richotte, Lusignan, Beauharnois, Hocquart, Varin. f 324, p. 181.

117-2. A la Baye, 4 juin 1745. «Memoire des fournitures de vivres faites par nous fermiers de la Baye aux chefs Saquis et Renard, allant en paroles a Montreal, par ordre de Mr de Lusignan, Commandant pour le Roy au Poste. » (Diverses fournitures.) Signé Richotte et Compagnie, Lusignan, Beauharnois, Hocquart, Varin. f 354, p. 214.

115-1. Camp devant Tournay, 15 juin 1745. « Pour les Depenses de Canada a payer du fonds du Domaine D'occident de l'année 1744 (sic). » (Officiers de guerre et garnisons entretenues; Québec, Montréal, Trois-Rivières ; maisons religieuses : Québec, Montréal ; officiers de Justice et de Police : Québec, Montréal, Trois-Rivières ; hôpital de Québec; dépenses extraordinaires; dépenses de l'Isle Royale; dépenses de la Louisiane; récapitulation.) f 146-149, pp. 385-396. 
115-2. Camp sous Tournay, 15 juin 1745. «Estat des Payemens que le Roy veut et ordonne estre faits par Me Marcellin françois Zacharie de Selle Trésorier général de la Marine pour les Depenses mentionnées cy après, faites et a faire pour le Service de Sa Majesté au pays de Canada pendant l'année 1745. (Construction des bateaux et radoub des canots ; courses et voyages; fret et voitures ; journées d'ouvriers et façons d'ouvrages; achats de marchandises, présents a faire aux Sauvages; bois a brûler ; subsistance et remè' des; dépenses imprévues ; appointements des officiers généraux et autres ; appointements des officiers reformés ; solde des compagnies ; gages et entretien des employés dans les magasins et bureaux : Québec, Trois-Rivières, Montréal ; gratifications ordinaires, extraordinaires : Québec, Montréal, Trois-Rivières; autres diverses dépenses; fortifications ; réparations ; récapitulation.) f 229-235, pp. 164178.

117-1. Montréal, 18 juillet 1745. «Memoire des fournitures que j’ai faites aux Ponteouatamis et autres Nations du Poste de la Rivière St Joseph au refus de Messieurs Les fermiers dud Poste. » (Diverses fournitures, 1742 et 1743). Signé de Villiers, Beauhamois, Hocquart, Varin. f 325 , pp. $182 \cdot 183$.

117-2. Détroit, 31 juillet 1745. «Memoire dés petites fournitures que j'ay faite pour le Roy, par L'ordre de Monsieur le Comman' dant.» (Diverses fournitures.) Signé Louis Campeau, Longueuil, Navarre, Beauharnois, Hocquart, Varin. f 355, p. 218.

117-2: Détroit, 4 août 1745. «Certificat pour Poitevin pour avoir Tiré des Boetes. ». (Certificat.) Signé Longueuil, Beauharnois, Hocquart, Varin. f 369 , p. 234.

117-1. Miamis, 16 août 1745. «Certificat de fourniture pour Lan, guedoc. » (Certificat.) Signé Douville, Beauharnois, Hocquart, Varin. f 351 , p. 214.

117-2. Miamis, 18 août 1745. « Certificat de livraisons de marchandises pour Parent. 》. (Certificat et fournitures.) Signé Raimbault, Longueuil, Beauharnois, Hocquart, Varin. f 372, p. 235.

117-2. Michilimackinac, 27 août 1745. «Certificat pour livraison d'un esclave. » (Certificat et fourniture.) Signé La Corne, La Ga' lissionière, Hocquart, Varin. f 421, p. 286. 
117-1. Détroit, 26 août 1745. «Memoire des Ouvrages que j’ay moy Belle perche par Lordre de Monsieur de Longueil pour les françois qui vont a Niagara pour le service du Roy du 25 aoust 1745. » (Diverses foumitures.) Signé Belleperche, Longueuil, La Galissionière, Hocquart. f 137-138, pp. 252-254.

117-2. Michilimackinac, 5 septembre 1745. «Mémoire des fournitures que jais fait au Roy par ordre de $\mathrm{M}$ de lacorne capitaine $\mathrm{Et}$ commandant pour Sa Majesté a Missilimakinac pour l'execution des ordres de Monsieur Le General. » (Certificat et fournitures.) Signé Disiers, La Corne, Beauharnois, Varin. f 374, p. 239.

118. Michilimackinac, 25 septembre 1745. «Memoire des foumitures faites par moy Thomas Blondeau pour la guerre ordonnée par Monsieur le Général par ordre de Monsieur de La Corne Capitaine et Commandant pour le Roy au Poste de Missilimakinac. » (Diverses fournitures.) Signé Thomas Blondeau, Beauharnois, Hocquart, Varin. f 45, p. 62.

117-1. Montréal, 31 décembre 1745. «Préparatifs de Guerre 3 derniers mois de 1745. \(Munitions ; marchandises ; vivres, sommaire.) Signé Hocquart, Varin. f 81-82, pp. 145-148.

117-1. Québec, 26 octobre 1746. «Preparatifs de Guerre 17.45 et 1746. » (Achats de munitions, 1745 et 1746.) Signé Hocquart, Va. rin. f 49-67, pp. 71-115.

117-1. Québec, 26 octobre 1746. «Preparatifs de Guerre 1745 et 1746. » (Achats de vivres, 1745 et 1746). Signé Hocquart, Varin. f 68.74 , pp. 116-131.

117-1. Québec, 26 octobre 1746. «Préparatifs de guerre 1745 » (Artillerie.) Signé Hocquart, Varin. f 87-89, pp. 156-161.

115-1 et 115-2. Québec, 18 septembre 1747. «Bordereau des Recettes et Dépenses faites par le Sr Taschereau Commis en Canada de Mrs Les Tresoriers généraux de la Marine pendant l'année 1745 ainsi qu'il en Suit. » (Recettes : fonds ordonnés par l'état du Roy ; déduction des dépenses de 1743 et 1744 ; recettes extraordinaires : des Ecclsésiastiques de Montréal ; imposition sur les bourgeois de Montréal ; produit des congés; produit des Postes des pays d'en haut ; ventes des magasins : Québec, Trois-Rivières, Montréal ; total 
de la recette. Dépenses : construction et radoub de bateaux et canots : Québec, Trois-Rivières, Montréal ; courses et voyages : Québec, Trois-Rivières, Montréal ; fret et voiture : Québec, Trois-Rivières, Montréal ; journées d'ouvriers et façons d'ouvrages : Québec, Trois-Rivières, Montréal ; achats de marchandises : marchandises et munitions: Québec, Trois-Rivières, Montréal ; vivres: Québec, Trois-Rivières, Montréal, Frontenac, Niagara ; bois a brûler : Qué bec, Trois-Rivières, Montréal ; hôpitaux : Québec, Trois-Rivières, Montréal ; dépenses imprévues : Québec, Trois-Rivières, Montréal, Chambly, St. Fréderic, Frontenac, Niagara ; appointements des offciers généraux et autres ; appointements des officiers reformés ; solde des compagnies ; gages et entretien des employés dans les magasins et bureaux : Québec, Trois-Rivières, Montréal ; gratifications ordinaires, extraordinaires : Québec, Trois-Rivières, Montréal ; autres diverses dépenses; fortifications et réparations : fortifications: Québec ; réparations : Québec, Trois-Rivières, Montréal, Chambly, Frontenac, Niagara, St. Fréderic; artillerie: Québec, Montréal ; total de la dépense. A déduire les sommes des états de 1746, 1747 et 1748.) Signé Hocquart, Varin. f 150-170, pp. 397-460, (vol. 115-1) et f 171-228, pp. 1-163, (vol. 115-2).

117-1. Québec, 18 septembre 1747. «Reparations aux fortifications Et Bâtimens Civils 1745. 》 (Pour le parfait payement des dépenses de l'année 1745.) Signé Hocquart, Varin. f 156, p. 285.

115-2. Québec, 30 octobre 1748. «Memoire au Sujet de la Situation des Finances de Canada de L'année MVII' quarente cinq. » (Fonds ordonnés par l'état du Roy ; fonds ordonnés par l'état du Roy pour 1746 ; fonds ordonnés par l'état du Roy pour 1747 ; fonds ordonnés par l'état du Roy pour 1748 ; récapitulation. Dépenses de 1745 ; récapitulation.) Signé Bigot. f 250-253, pp. 204 216.

115-2. Québec, 25 octobre 1750. «Bordereau des acquits des Re cettes et Depenses Employés dans le Compte de l'Exercice de mil sept cent quarante cinq. 》 (Recettes : fonds ordonnés par l'état du Roy; imposition de Montréal ; prix de les Fermes des Postes des pays d'en haut; ventes des magasins: Québec, Trois-Rivières, Montréal. Dépenses : construction et radoub des bateaux et canots : Québec, Montréal ; courses et voyages: Québec, Trois-Rivières, 
Montréal ; fret et voiture : Québec, Trois-Rivières, Montréal ; journées d'ouvriers et façons d'ouvrages : Québec, Trois-Rivières, Montréal ; achats de marchandises et munitions: Québec, Montréal ; achats de vivres : Québec, Trois-Rivières, Montréal ; bois a brûler : Québec, Trois-Rivières, Montréal ; hôpitaux : Québec, Trois-Rivières, Montréal ; dépenses imprévues : Québec, Trois-Rivières, Montréal, Frontenac, Niagara, St. Fréderic ; appointements des officiers généraux et autres ; appointements des officiers reformés ; appointements et solde des troupes : Québec, Trois-Rivières, Montréal ; appointements et gages d'employés : Québec, Trois-Rivières, Montréal, Frontenac, Niagara ; loyers de maisons : Québec, Montréal ; gratifications ordinaires, extraordinaires; autres dépenses. Deuxième chapitre de dépenses : fret et voiture : Montréal ; achats de mar chandises et munitions : Québec, Montréal ; achats de vivres : Montréal ; dépenses imprévues : St. Fréderic; appointements et gages d'employés ; fortifications ; réparations ; Trois-Rivières, Frontenac ; autres dépenses. Troisième chapitre de dépenses : achats de mar chandises et munitions : uébec; achats de vivres: Québec, Montréal ; fortifications : Québec, Montréal ; réparations : Québec, Niagara ; artillerie : Québec, Montréal ; sommaire du présent bordereau : dépenses 1745, 1744, 1743.) Signé Bigot, Imbert. f 236-249, pp. 179-208.

Voir aussi : 1746. 117-2. St. Joseph, 13 mai 1746. « Memoire des Marchandises... 》f 461, pp. 336-337.

Voir aussi : 1746. 117-2. St. Joseph, 14 mai 1746. « Memoire... 》 f 463, p. 339.

Voir aussi : 1744. 115-1. Québec, 25 septembre 1746. «Bordereau... $1744 \ldots \gg$ f 118 , p. 326.

Voir aussi : 1746. 117-2. Miamis, 6 juin 1747. « Memoire... armurier.. » f 367, p. 231.

Voir aussi : 1743. 115-1. Québec, 15 octobre 1748. « Memoire... » f 61, p. 142.

Voir aussi : 1744. 116-1. Québec, 30 octobre 1748. « Memoire... MVIIc quarante quatre... »f 83-86, pp. 129-136. 
Voir aussi : 1746. 116-1. Québec, 31 octobre 1748. «Memoire... MVII' quarante six. »f 88,90, pp. 138-143.

Voir aussi : 1748. 116-1. Québec, 1 novembre 1748. «Bordereau... acquits... MVIIc Quarante huit. »f 122-125, pp. 215-221.

Voir aussi : 1749. 1161. Québec, 3 novembre 1748. « Extraits des fonds a ordonner... »f 141, p. 255.

Voir aussi : 1747. 115-2. Québec, 3 novembre 1748. «Memoire... 》f 365 , p. 412.

Voir aussi : 1746. 115-2. Québec, 24 octobre 1751. «Bordereau général... 1746. 》 f 324-327, pp. 338-340.

Voir aussi : 1746. 115-2. Québec, 15 août 1752. «Bordereau... $1746 \ldots$... 280 , p. 261.

Voir aussi : 1747. 116-1. Québec, 20 septembre 1752. «Borde reau... 1747. »f 36 , p. 66-67.

\section{6 \\ C $11 \mathrm{~A}$}

118. St. Joseph, 4 janvier 1746. «Fourniture dun cheval par Char, les Marchand. » (Certificat.) Signé de Blainville, Beauharnois, Hocquart, Varin. f 20, p. 29.

118. A la Grande Rivière, 15 janvier 1746. «Certificat. 》 (Fournitures.) Signé La Corne, Beauharnois, Hocquart, Varin. f 50, p. 68.

118. A la Grande Rivière, 26 janvier 1746. «Certificat. » (fournitures.) Signé La Corne, Beauharnois, Hocquart, Varin. f 39, p. 54.

118. Chicago, 10 février 1746. «Memoire des ouvrages que j’ay faits pour le Roy par ordre de $M$ de Villers pour les sauvages de Chikagou. » (Diverses ouvrages de forgeron.) Signé Amiot, de Villiers, Blainville, Beauharnois, Hocquart, Varin. f 7, p. 9.

117-2. St. Joseph, 23 février 1746. «Certificat. 》 (Fournitures.) Signé Blainville, Beauharnois, Hocquart, Varin. f 458, p. 353.

115-2. Versailles, 20 mars 1746. «Sur les fonds du Domaine d'oc' cident. Pour les Depenses de Canada a payer du fonds du Domaine 
d'occident de l'Année 1746. » Officiers de guerre et garnisons : Québec, Montréal, Trois-Rivières ; maisons religieuses : Québec, Montréal ; officiers de Justice et de Police: Québec, Montréal, TroisRivières ; autres officiers de Justice et de Police ; hôpital de Québec ; dépenses extraordinaires; dépenses de la Louisianne ; récapitulation.) f 301-304, pp. 303-312.

115-2. Versailles, 20 mars 1746. «Estat des Depenses que le Roy veut et ordonne estre faits par Mr Louis Barthelemy Moufle de Georvile Tresorior géneral de la Marine pour les Dépenses mentionnées cy après faites et à faire pour le Service de Sa Majesté au Pays de Canada pendant l'année 1745 (sic). ». (Construction et radoub de bateaux et canots; courses et voyages; fret et voiture ; journées d'ouvriers et façons d'ouvrages ; présents a faire aux Sauvages ; bois a brûler ; subsistance et remèdes; dépenses imprévues ; appointements des officiers généraux et autres; appointements des officiers reformés ; solde des compagnies ; gages et entretien des employés dans les magasins et bureaux ; gratifications ordinaires, extraordinaires : Québec, Montréal ; Trois-Rivières ; dépenses pour les préparatifs de guerre ; autres diverses dépenses ; fortifications ; réparations ; récapitulation.) f 331-337, pp. 346-359.

118. A la Grande Rivière, 5 avril 1746. «Memoire des fournitures faites par moy Louis Lefebvre forgeron pour la guerre a la grande Riviere par ordre de $\mathrm{M}$ de La Corne cap'ne et Commandant pour le Roy au poste de Missilimakinac. » (Diverses ouvrages et fournitures.) Signé Lefebvre, La Corne, Beauharnois, Hocquart, Varin. f 27, pp. 37-38.

118. Détroit, 25 avril 1746. «Certificat.» (Fournitures.) Signé Longueuil, Navarre, Beauharnois, Hocquart, Varin. f 58, p. 76.

117-2. St. Joseph, 4 mai 1746. «Certificat. » (Fournitures.) Signé Madame de la Perrières, Blainville, Beauharnois, Hocquart, Varin. f 462 , p. 338.

118. St. Joseph, 4 mai 1746. «Memoire de ce que j'ay fourni pour le service du Roy par ordre de M Blainville pour faire festin aux Pouteouatamis qui descendent pour la guerre. \ (Diverses foumitures.) Signé de Quindre, Blainville, Beauharnois, Hocquart, Varin. f 8, p. 10 . 
118. St. Joseph, 8 mai 1746. «Memoire des fournitures faites pour le Roy quant les Pouts et les autres Nations de St. Joseph ont com' mencé a chanter la guerre le 157 'bre par l'ordre de $M$ de Blainville commandant pour le Roy au poste de St. Joseph par moy Jean Baptiste Dubois Interprette dud. Poste. » (Diverses fournitures.) Signé Blainville, Beauharnois, Hocquart, Varin. f 6, p. 8.

117-2. St. Joseph, 13 mai 1746. « Certificat. » (Fourniture.) Signé Blainville, Beauharnois, Hocquart, Varin. f 459, p. 334.

117-2. St. Joseph, 13 mai 1746. «Memoire des Marchandises que nous avons fournies pour le compte du Roy par ordre de $\mathrm{Mr}$ de Blainville depuis le 15 novembre 1745 jusquau 13 may 1746. » (Diverses fournitures.) Signé de Quindre, La Perrière Marin, Blainville, Beauharnois, Hocquart, Varin. f 461, pp. 336-337.

117-2. St. Joseph, 14 mai 1746. «Memoire de ce que nous avons fourniy pour le Roy par ordre de Mr de Blainville depuis le 29 octo bre jusqu'au 13 may 1746. » (Diverses fournitures.) Signé de Quin. dre, La Perrière Marin, Blainville, Beauharnois, Hocquart, Varin. f 463, p. 339.

117-2. St. Joseph, 15 mai 1746. «Memoire des fournitures que moi Deshaitre a fait pout le Roy par ordre de Mon's de Blainville. » (Diverses fournitures.) Signé Deshaitre, Blainville, Beauharnois, Hocquart, Varin. f 452, p. 326.

117-2. St. Joseph, 15 mai 1746. «Memoire de ce que j’ay fourny au Roy par l'ordre de $\mathrm{Mr}$ de Blainville officier Commandant pour le Roy au Poste de St. Joseph. ». (Diverses fournitures.) Signé Veuve Larche, Blainville, Beauharnois, Hocquart, Varin. f 454, p. 328.

117-2. St. Joseph, 15 mai 1746. «Memoire des fournitures faites par moy dehaitre par ordre de Monsieur de Blainville officier des Troupes du Détachement de la Marine et Commandant pour le Roy au poste de St Joseph pour l'Execution des ordres de Monsieur le Général a L'occasion des Lévées des Pont8atamis. \ ${ }^{5}$ (Diverses fournitures; gages d'employés.) Signé Deshaitre, Beauharnois, Blainville, Hocquart, Varin. f 464, p. 340 .

5. Le 8 représente les lettres « ou . 
117-2. Michilimackinac, 21 mai 1746. «Estat de ce que j'ay fourny pour le Compte du Roy a Mr de Villiers. » (Etat de fourniture.) Signé LaMothe d'Ouville, La Corne, Beauharnois, Varin. f 375, p. 240.

118. Poste de la Baye, 26 mai 1746. «Estat des marchandises four nis Nous fermiers de la Baye par ordre de Mr de Lusignan Com: mandant pour le Roy aud. Poste pour parler aux Nations en execution des ordres de Monsieur le Général pour la guerre. » (Diverses fournitures.) Signé C. Texier et Compagnie, Lusignan, Beauharnois, Hocquart, Varin. f 3-5, pp. 1-7.

118. Détroit, 27 mai 1746. «Certificat. » (Fourniture.) Signé Longueuil, Navarre, Beauharnois, Hocquart, Varin. f 55, p. 73.

118. Détroit, 28 mai 1746. «Certificat. » (Fourniture.) Signé Longueuil, Navarre, Beauharnois, Hocquart, Varin. f 52, p. 70.

118. Détroit, 29 mai 1746. «Fournitures faites par moy Jacques Campeau. » (Diverses fournitures.) Signé Longueuil, Navarre, Beauharnois, Hocquart, Varin. f 60 , pp. 78,79.

118. Michilimackinac, 20 mai 1746. «Memoire des fournitures que j'ay faites par l'ordre de $\mathrm{Sr}$ de la Corne Capitaine et Commandant pour le Roy en ce poste pour la descente des Sauvages a Montreal pour la guerre. » (Diverses fournitures.) Signé Dubois, La Cornes, Beauharnois, Hocquart, Varin. f 16, p. 25.

118. Michilimackinac, 30 mai 1746. «Certificat. » (Fourniture.) Signé Dubois, Blainville, Beauharnois, Hocquart, Varin. f 21, p. 30.

118. Michilimackinac, 1 juin 1746. «Memoire de ce que moy Pierre Monbront ait fait pour La Guerre ordonné par Monsieur Le Général par ordre de $\mathrm{M}$ de la Corne capitaine Commandant pour le Roy a Missilimakinac. » (Diverses fournitures.) Signé Monbront, La Corne, Beauharnois, Hocquart, Varin. f 17, p. 26.

117-2. St. Joseph, 3 juin 1746. «Memoire de ce que j’ay fourni pour le Roy par L'ordre de $\mathrm{Mr}$ de Blainville Commandant en ce Poste. » (Diverses fournitures.) Signé : de Villiers, la Perrière, Blainville, Beauharnois, Hocquart, Varin. f 460, p. 335. 
118. Michilimackinác, 3 juin 1746. «Memoire de ce que moy Des carie ay fourny pour la Guerre aux departs des.Pouts et Illinois a missilimakinac a Mon's de Blainville Command't de St. Joseph pär ordre de monsieur le marquis de Beauharnois et Livré par ordre de m. de La corne cap'ne et Commandant a Missilimakinac. » (Fournitures.) Signé Décarie, La Corne, Beauharnois, Hocquart, Varin. f 44, p. 61 .

118. Détroit, 3 juin 1746: «Certificat. 》. (Fourniture.) Signé Longueuil, Navarre, Beauharnois, Hocquart, Varin. f 51, p. 69.

117-1. Michilimackinac, 4 juin 1746. «Memoire de ce que le fils de Louis Lameigue Nouiakousye, Pontouatamis a fourny pour le Roy.» (Fournitures.). Signé Blainville, La: Corne, Beauharnois, Hocquart, Varin. f 352, p. 215.

117-2. Détroit, 5 juin 1746. «Certificat de fourniture de canot pour Cueillerier. » (Certificat.) Signé Longueuil, Navarre, Beauharnois, Hocquart, Varin. f 373 , p. 238.

117-2. Miamis, 6 juin 17.46. « Memoire dés ouvrages que monsieur le Commandant a fait faire par Barthe Armurier aux miamis pour le compte du Roy.» (Diverses ouvrages). Signé Barthe, Douville, Beauharnois, Hocquart, Varin. f 367 ; pp. 231-232.

117-2. Michilimackinac; 6 juin 1746 « Memoire que j’ay fourny par ordre de Monsieur de Lacorne Capitaine d'une Compagnie des Troupes commandant pour le Roy a Missilimakinac pour la descente des sauvages a Montréal suivant les ordres de Monsieur le Géné ral. » (Fourniture.) Signé Jasmin, La Corne; Beauharnois, Hocquart, Varin. f 451, p. 325.

118. Détroit, 6 juin 1746. «Certificat. » (Fourniture:) Signé Lon' gueuil, Navarre, Beauharnois, Hocquart, Varin. f 57, p. 75.

118. Michilimackinac, 7 juin 1746. «Memoire des fournitures faites par moy Jean Baptiste Descarie a la Riviere a la Roche a Monsieur de Villier officier pour l'Execution des ordres de Monsieur le Général a l'occasion de la Levée des Nations pour la Guerre.:» (Diverses fournitures.) Signé Jean Décarie, Lusignan, Beauharnois, Hocquart, Varin. f 47, p. 74. 
117-2. Michilimackinac, 8 juin 1746. «Certificat. 》. (Fourniture.) Signé La Corne, Beauharnois, Hocquart, Varin. f 447, p. 318 :

118. Michilimackinac, 11 juin 1746. «Memoire des fournitures que j'ay faites au Roy par l'ordre de $\mathrm{M}$ de la Corne Cap'ne Comman. dant En ce poste pour le Roy pour faire les presens aux nations qui vont a Montreal pour la guerre. » (Diverses fournitures:) Signé Giasson, La Corne, Beauharnois, Hocquart, Varin. f 28, p. 39.

118. Michilimackinac, 12 juin 1746. «Memoire des fournitures que j'ay faites au Roy par l'ordre de Monsieur de La Corne Capitaine Commandant pour sa Majesté a Missilimakinac pour l'Execution des ordres de Monsieur le Général. » (Diverses fournitures.) Signé Ducharme, La Corne, Beauharnois, Hocquart, Varin. f 22, p. 31.

118. Michilimackinac, 12 juin 1746. «Memoire de ce que moy jean Baptiste Chevalier ay fourny pour la Guerre ordonnée par Monsieur le Général par ordre de $\mathrm{M}$ de la Corne cap'ne Commandant pour le Roy a Missilimakinac. » (Fourniture.) Signé Chevalier, La Corne, Beauharnois, Hocquart, Varin. f 29, p. 40.

118. Michilimackinac, 12 juin 1746. « Memoire des fournitures que j'ay faites au Roy par ordre de Mr de Lacorne cap'ne Commandant pour le Roy en ce Poste pour le depart des Sauvages pour la guerre a Mont'el. » (Diverses fournitures.) Signé Giasson, La Corne, Beauharnois, Hocquart, Varin. f. 36, p. 50.

117-1. Michilimackinac, 15 juin 1746. «Certificat de fourniture pour Kinonchameque. » (Certificat.) Signé La Corne, Michel, Beauharnois, Hocquart, Varin. f 353, p. 216.

118. Michilimackinac, 16 juin 1746. «Certificat. 》 (Fourniture.) Signé de Villiers, La Corne, Beauharnois, Hocquart, Varin. f 31 , p. 44.

118. Michilimackinac, 18 juin 1746. « Memoire des fournitures que j'ay fait au Roy par L'ordre de Monsieur de la Corne Capitaine et Commandant pour le Roy en ce Poste pour l'Execution des ordres de Monsieur le Général. » (Diverses fournitures.) Signé N. Volant, La Corne, Beauharnois, Varin. f 40, pp. 55,56.

118. Michilimackinac, 19 juin 1746. « Memoire des fournitures que j'ay faites au Roy par ordre de $\mathrm{M}$ des Noyelle officier Commandant 
en Second a Missilimakinac pour Sa majesté de presens au saguinente pour L'Execution des ordres de monsieur Le General pour la guerre. » (Diverses fournitures.) Signé Giasson, La Corne, Beauharnois, Hocquart, Varin. f 35 , pp. 48-49.

118. Michilimackinac, 21 juin 1746. «Memoire des fournitures que j'ay faites au Roy par ordre de Monsieur de La Corne Cap'ne Com. mandant pour le Roy en ce poste pour le depart des Sauvages pour aller a Montreal pour la guerre. » (Diverses fournitures.) Signé Jean Lefebvre, La Corne, Beauharnois, Hocquart, Varin. f 24, p. 33.

118. Michilimackinac, 22 juin 1746. «Memoire des fournitures faites par moy Charles Thomas Blondeau pour la guerre ordonnée par Monsieur Le Général par ordre de Monsieur de La Corne Capitaine et Commandant pour le Roy au poste de Missilimakinac. » (Diverses fournitures.) Signé Thomas Blondeau, La Corne, Beauharnois, Hocquart, Varin. f 23, p. 32.

118. Michilimackinac, 23 juin 1746. «Memoire de ce que moy Nicolas Dufresne a fourny pour la guerre ordonnée par Monsieur le Général pour le service de Sa Majesté et par ordre de Monsieur de La Corne Capitaine et Commandant pour le Roy a Missilimakinac. » (Diverses fournitures.) Signé Nicolas Dufresne, La Corne, Beauharnois, Hocquart, Varin. f 25, pp. 3435.

118. Michilimackinac, 23 juin 1746. «Memoire des fournitures que j’ay faites au Roy par ordre de Mr Lacorne capitaine Commandant pour le Roy en ce Poste pour faire les presens aux Sauvages pour descendre a Montreal pour la guerre. » (Diverses fournitures.) Signé Disier, La Corne, Beauharnois, Hocquart, Varin. f 34, p. 47.

118. Michilimackinac, 23 juin 1746. « Memoire des fournitures que j'ay faites au Roy par ordre de $\mathrm{M}$ de Lacorne cap'ne Commandant pour sa majesté en ce Poste pour l'Execution des ordres de M Le general. »(Diverses fournitures.) Signé Ignace Hubert, La Corne, Beauharnois, Hocquart, Varin. f 37-38, pp. 51-53.

117-2. Michilimackinac, 24 juin 1746. «Memoire des fournitures que j'ay faites au Roy par ordre de $\mathrm{Mr}$ De la Corne Capitaine Com. mandant de ce poste pour le départ des sauvages qui vont a Montreal pour La Guerre. » (Diverses fournitures.) Signé Joseph Saint-Germain, La Corne, Beauharnois, Hocquart, Varin. f 445, p. 316. 
117-2. Michilimackinac, 25 juin 1746. «Memoire des fournitures que j'ay faites au Roy Livrées a Monsieur de Noyelles officier en second Commandant pour le Roy a Missilimakinac. ». (Diverses fournitures.) Signé : Disier, La Corne, Beauharnois, Hocquart, Varin. f 455, p. 329.

117-2. Michilimackinac, 25 juin 1746. «Mémoire des fournitures que j'ay faites au Roy par L'ordre de $\mathrm{M}$ de la Corne cap'ne Com. mandant pour sa Majesté en ce poste pour fournir aux sauvages le Tems qu'ils ont été icy que pour Leur descente a Montréal pour la guerre. » (Diverses fournitures.) Signé Pierre Auvoy dit Duchesne, $\mathrm{La}$ Corne, Beauharnois, Hocquart, Varin. f 456, pp. 33-331.

117-2. Michilimackinac, 25 juin 1746. «Mémoire des fournitures que j’ay faites au Roy par L'ordre de M de la Corne cap'ne Com. mandant pour sa Majesté en ce poste pour fournit aux sauvages le Tems qu'ils ont été icy pour Leur descente à Montréal pour la guerre. » (Diverses fournitures.) Signé Pierre Auvoy dit Duchesne, La Corne, Beauharnois, Hocquart, Varin. f 456, pp. 330-331.

118. Michilimackinac, 25 juin 1746. «Memoire de ce que moy Jean Baptiste Joliet ai fourni pour La Guerre ordonnée par Monsieur Le Général pour le Service du Roy et par ordre de Monsieur de La Corne Capitaine Commandant pour Le Roy a Missilimakinac. » (Diverses fournitures.) Signé La Corne, Beauharnois, Hocquart, Varin. f 43 , pp. $59 \cdot 60$.

118. Michilimackinac, 25 juin 1746. «Memoire des fournitures que j'ay faite au Roy par l'Ordre de Monsieur de La Corne Capitaine et Commandant pour sa Majesté a Missilimakinac. » (Diverses four nitures.) Signé Joseph Décarie, La Corne, Beauharnois, Hocquart, Varin. f 46, p. 63.

117-2. Michilimackinac, 30 juin 1746. «Memoire fait par moy Louis Lefebvre forgeron de Missilimakinac pour la Guerre par ordre de $\mathrm{M}$ de Lacorne cap'ne et Commandant pour le Roy au poste de Missilimakinac. » (Diverses ouvrages et fournitures.) Signé Louis Lefebvre, La Corne, Beauharnois, Hocquart, Varin. f 448-450, pp. 319-324.

118. Michilimackinac, 2 juillet 1746. «Memoire des fournitures faites par moy Louis Hurtubise pour la guerre par ordre de Mon- 
sieur de La corne Capitaine Commandant pour le Roy au poste de Missilimakinac. » (Diverses fournitures.) Signé Louis Hurtubise, $\mathrm{La}$ Corne; Beauharnois, Hocquart, Varin. f 26, p. 36.

118. Michilimackinac, 3 juillet 1746. «Memoire des fournitures que j'ay faites au Roy par lordre de $\mathrm{M}$ de la Corne capitaine et Commandant pour le Roy en ce poste. i). (Diverses fournitures.) Signé Laplante, Bourassa, La Corne, Beauharnois, Hocquart, Varin. f 30 , pp. $41 \cdot 43$.

118. Michilimackinac, 5 juillet 1746 : « Memoire des fournitures faites par moy François Brandsard, dit Langevin pour la Guerre par ordre de Monsieur de $\mathrm{La}$ Corne Capitaine et Commandant pour le Roy au poste de Missilimakinac. »' (Diverses fournitures.) Signé Brandsard, La Corne, Beauharnois, Hocquart, Varin. f 42, p. 58.

118. Michilimackinac, 5 juillet 1746. «Memoire des fournitures faites par moy Gabriel Bollay pour la Guerre a Monsieur de Blainville officier et Commandant pour le Roy au poste de St. Joseph et par ordre de Monsieur de La Corne au poste de Missilimakinac. » (Diverses fournitures.) Signé Gabriel Bollay, La Corne, Beauhar. nois; Hocquart, Varin. f 48, p. 65.

118. Michilimackinac, 6 juillet 1746 . « Certificat. »' (Fourniture.) Signé La Corne, Beauharnois, Hocquart, Varin. f 41, p. 57.

118. Montréal, 6 juillet 1746. « Mémoire de ce que jay fourny a deux jeunes gens Iroquois du Lac des deux Montagnes Envoyés aux Cinq Nations pour y penetrer leur desseins; a find de rendre compté a Monsieur Le General. » (Diverses fournitures.) Signé Piquet prêtre, Beauharnois, Hocquart, Varin. f 63 , p. $84:$ :

118. Montréal, 20 juillet 1746 . Memoire de la depense que j’ay. fait lorsque les sauvages des Cinq nations sont arrivés au Lac des deux Montagnes ou ils ont séjournés trois jours venant de leur pays a Montreal et a leur retour y ayant aussy: séjournés trois jours, et lorsque les Outa8acs, Les Mississagués, et les nations du Nord sont descendues et se sont arrestés au Lac, pour ét a L'occasion de lá guerre. » (Diverses fournitures.) Signé Piquet prêtre; Beauharnois; Hocquart, Varin. f 62, pp. 82-83.

118. Détroit, 24 juillet 1746. «Certificat ». (Fourniture.) Signé Longueuil, Navarre, Beauharnois, Hocquart, Varin. f 56, p. 74. 
117-1. Détroit, 25 juillet 1746. «Certificat de gages pour Poitevin ayant servi comme Canonnier. » (Certificat de service et gage.) $\mathrm{Si}$ gné Longueuil, Beauharnois, Hocquart, Varin. f 350, p. 213 :

118. Détroit, 25 juillet 1746. «Certificat. 》 (Fourniture.) Signé Longueuil, Navarre; Beauharnois, Hocquart, Varin. f 53, p. 71.

118. Détroit, 25 juillet 1746. «Certificat.» (Fourniture.) Signé Longueuil, Navarre, Beauharnois, Hocquart, Varin. f 59, p. 77.

118. Détroit, 25 juillet 1746. «Memoire des fournitures faites par moy Antoine Cuillerier au Détroit pour le compte et 'service du Roy par les ordre de $\mathrm{Mr}$ Ch'er de Longueuil Commandant pour le Roy aud. Lieu comme cyraprès. » (Diverses fournitures.) Signé Cuillerier, Longueuil, Navarre, Hocquart, Varin. f 61, pp. 80-81.

117-2. Détroit, 26 juillet 1746. «Certificat de fourniture de canot pour Antoine Cuillerier. » (Certificat.) Signé Longueuil, Navarre, Beauharnois, Hocquart, Varin. f 372, p. 237.

117-2. Michilimackinac, 2 août 1746. «Certificat. 》 (Fourniture.) Signé Legras et Compagnie, La Corne, Beauharnois, Hocquart, Varin. f 446, p. 317.

118. Michilimackinac, 2 août 1746. «Memoire des fournitures que moy mongras ay faites pour le Roy par l'ordre de $\mathrm{Mr}$ de Lacorne Commandant a Missilimakinac suivant les ordres de Monsieur le Général pendant mon absence. » (Diverses fournitures:) Signé La Corne, Beauharnois, Hocquart, Varin. f 32, p. 45.

119. Michilimackinac, 3 août 1746. «Memoire de ce j'ay fourni pour le Roy... a St Joseph. » (Diverses fournitures.) Signé Blainville, $\mathrm{La}$ Jonquière, Bigot. f 34, p. 42.

118. Michilimackinac, 4 août 1746. « Memoire des fournitures que j’ay faites au Roy par l'ordre de Monsieur de La Corne Capitaine et Commandant pour le Roy en ce poste pour conduire les Scioux ches eux. » (Diverses fournitures.) Signé Jean-Baptiste Lefebvre, $\mathrm{La}$ Corne, Beauharnois, Hocquart, Varin. f 33, p. 46.

117-2. Détroit, 10 août 1746. «Certificat de requête pour Cueilleriers commis du sieur Charly; pour fournitures de grains de Porce; laine. » (Certificat.) Signé Douville, Hocquart, Varin. f 376, p. 241. 


\section{L'ACTUALITÉ ECONOMIQUE}

117-2. Détroit, 22 août 1746. «Certificat pour Cardinal pour avoir amené des ouyas au Detroit. » (Certificat.) Signé de Lino, Beauharnois, Hocquart, Varin. f 368, p. 233.

117-2. Michilimackinac, 22 août 1746. «Memoire des fournitures que moy René Provanché et faites a Monsieur de Noyelle Commandant pour le Roy a Missilimakinac. » (Fourniture.) Provanché, Noyelles fils, La Galissionière, Hocquart, Varin. f 401, p. 266.

117-2. Détroit, 24 août 1746. «Certificat de fourniture pour $\mathrm{Pa}$ rent. » (Diverses fournitures.) Signé Longueuil, Beauharnois, Hoc' quart, Varin. f 371, p. 236.

118. St. Joseph, 27 août 1746. « Certificat. 》 (Diverses fournitures.) Signé François Beauchemin, Blainville, Sanschagrin, Hocquart. f 87, pp. 106-107.

118. Détroit, 29 août 1746. «Certificat. 》 (Fourniture.). Signé Longueuil, Navarre, Beauharnois, Hocquart, Varin. f 54, p. 72.

117.1. Montréal, 1 septembre 1746. «Préparatifs de Guerre 8 premiers mois 1746. \ (Munitions et marchandises ; vivres ; sommaire.) Signé Varin, Hocquart. f 83-86, pp. 149-155.

115-2. Montréal, 30 septembre 1746. «Estat de la depense qui a été faite a Montréal pour et a l'Occasion du party de françois et Sauvages Commandé par $\mathrm{M}$ de Rigaud de Vaudreuil major de la place des Trois Rivières pour aller sur les Costes de la Nouvelle Angleterre party de Cette Ville Le Trois Aoust 1746 ainsy quil Ensuit. 》 (Vivres et equipement pour la campagne.) Signé Martel, Michel. f 254-276, pp. 217-255.

117-1. Michilimackinac, 25 octobre 1746. «Memoire des fournitures que moy Duchêne a fait pour le Roy par ordre de Monsieur de Noyelle, officier et Commandant pour le Roy a Missilimakinac, a l'absence de Monsieur de La Corne. » (Diverses fournitures.) Signé Duchênes, Noyelles fils, Hocquart, Varin. f 335; pp. 197-198.

117-1. Québec, 26 octobre 1746. «Preparatifs de Guerre 1745 et 1746. » (Achats de munitions 1745 et 1746.) Signé Varin, Hocquart. f $49 \cdot 67$, pp. $71 \cdot 115$. 
117-1. Québec, 26 octobre 1746. «Preparatifs de Guerre 1745 et 1746. » (Achats de vivres 1745 et 1746.) Signé Varin, Hocquart. f 68-74, pp. 116-131.

117-1. Québec, 26 octobre 1746. «Preparatifs de guerre 1746. » (Caserne d'artifice ; lance de mer a l'Isle aux Coudres). Signé Varin, Hocquart. f 75, pp. 132-133.

117-1. Québec, 26 octobre 1746. «Preparatifs de guerre 1746. » (Chaloupes d’observations et de signaux.) Signé Varin, Hocquart. f 76, pp. 134136 .

117-1. Québec, 26 octobre 1746. «Armement pour L'accadie 1746. 》 (Effets pour navires; gages de l'équipages.) Signé Varin, Hocquart, f. 77-78, pp. 137-140.

117-1. Québec, 26 octobre 1746. «Armement pour L'Accadie. 》 (Appointements d'employés ; gratifications ; sommaire.) Signé Varin, Hocquart. f 79-80, pp. 141-144.

117-2. St. Joseph, 30 décembre 1746. «Memoire de ce que j’ai fourny pour le Roy au refus des fermiers et que j'ay ôté de ma provision. » (Diverses fournitures.) Signé Blainville, Beauharnois, Hoc' quart, Varin. f 453, p. 327.

115-2. Montréal, 31 décembre 1746. «Estat des payemens qui ont Eté ordonnés à Montréal pour les diverses Dépenses faites à l'occa: sion du Party de Guerre Commandé par Mr de Rigaud en 1746. » (Radoub d'armes ; de chaudières ; journées d'ouvriers employés dans les magasins ; remèdes et médicaments ; logements et pensions en ville et vivres dans les costes ; traverse et passage du fleuve ; courses et voyages; payements d'interprète ; aumônier ; autres dépenses.) Signé Varin, Hocquart. f 300, p. 302.

117-1. Montréal, 1 septembre 1747. «Depense a L'occasion de La guerre 4 d'ers mois 1746 Et 8 p'ers mois 1747.» (Etat des paye' ments qui ont été ordonnés à Montréal pour les diverses dépenses a l'occasion de la guerre pendant 1746 et 1747.) Signé Varin, Hocquart. f 150-151, pp. 274-276.

117.1. Québec, 10 octobre 1747. «Preparatifs de guerre 1746. 》 (Artillerie ; diverses dépenses de 1746 et 1747.) Signé Varin, Hocquart. f 148-149, pp. 270-273. 
117-1. Québec, 15 octobre 1747. «Dépenses a L'occasion de la guerre $17.46 \mathrm{Et}$ 1747. 》 (Dépenses faites : à diverses particuliers; diverses dépenses.) Signé Varin, Hocquart. f 147, pp. 268-269.

116-1. Québec, 31 octobre 1748. «Memoire au sujet de la situation des Finances du Canada de l'année MVIIc quarante six. » (Fonds ordonnés par l'état du Roy du 20 mars 1746 ; autres fonds ordonnés le 19 mars 1747.; autres fonds ordonnés le 24 février 1748 ; recettes extraordinaires; récapitulation; total de la recette. Dépenses de 1746 ; acquits non comprits au bordereau du 16 septembre 1748 ; récapitulation; balance.) Signé Bigot. f 87-90, pp. 137-143.

116-1: Québec, 24 octobre 1750. «Etat des fonds faits par les Etats du Roy du montant des Recettes Extraordinaires et des dépenses faites en Canada sur les Exercices de 1746, 47, 48 et 1749 pour servir à Constater les Excedents de Depenses de chacun de ces Exercices. 》(Exercice de 1746 : recettes : fonds faits par l'état du Roy pour 1746 ; fonds faits par l'état du Roy de 1747 pour les dépenses de 1746 ; fonds faits par l'état du Roy de 1748 pour les dépenses de 1746 ; recettes extraordinaires ; récapitulation. Dépenses : total de la dépense; sommäire.) Signé Bigot. f .238-245, pp. 409.424.

116-2. Québec, 26 octobre 1750. « Extrait du fonds par suplement pour le parfait payement des dépenses générales faites en Canada pendant l'année 1746. » (Dépenses : à déduire les fonds ordonnés ; fonds a ordonnés:) Signé Bigot. f :336; p. 209.

115-2. Québec, 24 octobre 1751. (Joint a la lettre de Bigot du 2 novembre 1751.) "Bordereau général de la Recette et Dépense faite en Canada pendant l'année 1746 dirigé suivant les Titres employés dans l'Etat du Roy Expedié sur cet Exercice. 》(Recettes extraordinaires : Québec, Trois-Rivières, Montréal. Dépenses : (les dépenses sont classifiées sous les titres suivant : dépenses de l'année; fonds ordonnés par l'état du Roy ; revenans bons ; excedents ; mo tifs.) construction et radoub de bateaux et canots; courses et voyages ; fret et voiture ; journées d'ouvriers et façons d'ouvrages ; achats de marchandises; bois a brûler ; subsistance et remèdes ; dépenses

6. Ce document se trouve àussi dans le présent inventaire pour les années 1747 et 1748 et 1749. Les années et les indications pour chaque année sont tellement peu claires qu'ont a cru bon suivre cette procédure. 
imprévues ; appointements des officiers généraux et autres ; appointements des officiers reformés ; solde des compagnies ; gages et entretien des employés dans les magasins; loyers de maisons et bureaux; gratifications ordinaires, extraordinaires : Québec, Montréal, Trois Rivières; fortifications; autres diverses dépenses; additions aux titres non compris dans l'état du Roy ; parti de guerre ; artillerie ; préparatifs de guerre pour les années 1747 et 1748 ; supplement de fonds pour les dépenses de 1746 tirés sur 1751.) Signé Bigot. f 314 330, pp. 325-348.

115-1. Québec, 15 août 1752. «Bordereau des Recettes et Dépen ses, Employées dans le Compte rendu à Ms Louis Barthelemy. Moufle de Georville, Tresorier général de la Marine pour lExercice 1746, par le Sr Imbert, faisant pour la Succéssion de feu M. Taschereau son Commis au Canada. » (Recettes : fonds ordonnés par l'état du Roy ; ventes des magasins du Roy : Québec, Trois-Rivières, Montréal ; total de la recette. Dépenses,: dépenses de 1744 : fonds faits dans l'état de 1746 ; dépenses de 1745 : fonds faits par l'état de 1746 ; dépenses de 1746 : construction et radoub de bateaux et canots : Québec, Montréal ; courses et voyages : Québec ; fret et voiture : Québec ; frets de barques et voitures de harnois : Montréal ; journées d'ouvriers et façons d'ouvrages : Québec, Montréal ; achats de marchandises et munitions : Québec, Montréal; achats de vivres: Québec, Montréal ; achats de bois a chaufage : Québec, Trois-Rivières, Montréal ; hôpitaux : Québec, Trois-Rivières, Montréal ; dépenses imprévues : Québec, Trois-Rivières, Montréal ; ap pointements et solde des troupes: Québec, Trois-Rivières, Montréal ; appointements et gages des employés : Québec, TroisRivières, Montréal, Frontenac, Niagara, St. Fréderic; loyers de maisons : Quẹbec, Montréal ; sommaire.) Signé Bigot. f 278-299, pp. 256-301.

115-2. Québec, 15 août 1752. « Bordereau des Dépenses Employés dans ladditions au Compte rendu a Monsieur de Georville Tresorièr général de la marine pour l'Exercice 1746. 》 (Construction et radoub de bateaux : Trois-Rivières; courses et voyages : Québec, Montréal ; fret et voiture : Québec, Montréal ; journées douviriers et façons d'ouvrages : Québec, Trois-Rivières, Montréal ; achats de marchandises : Québec, Trois-Rivières; Montréal, Frontenac, Niagara, St. Fréderic ; achats de vivres, Québec, Trois-Rivières, Mont- 


\section{L'ACTUALITE ECONOMIQUE}

réal ; achats de marchandises et vivres : St. Fréderic ; dépenses im. prévues : Québec, Trois-Rivières, Montréal, Chambly, Frontenac, Niagara, Sté Fréderic ; fortifications : Québec ; réparations : de 1744 et 1746 : batteries : Trois-Rivières, Montréal, Chambly, Frontenac, St. Fréderic ; hôpital : Montréal ; officiers reformés ; autres dépenses ; artillerie : Québec, Montréal, St. Fréderic.) Signé Bigot. f 339 . 363, pp. 361.409.

Voir aussi : 1747. 117-1. Québec, 26 octobre 1746. «Preparatifs de guerre 1746. »f 48, pp. 69-70.

Voir aussi : 1747. 117-1. Québec, 26 octobre 1746. «Dépenses... Exercice 1747. 》f 91-93, pp. 163-168.

Voir aussi : 1745. 115-2. Québec, 18 septembre 1748. "Bordereau... $1745 \ldots$... 227 , p. 162 .

Voir aussi : 1744. 115-1. Québec, 25 septembre 1747. «Bordereau... $1744 \ldots \gg$ f 118 , p. 326.

Voir aussi : 1747. 117-1. Québec, 15 octobre 1747. «Fort St. Frederic, 1746. 》 f 1-2, pp. 2-4.

Voir aussi : 1747. 117-1. Québec, 15 octobre 1747. «Preparatifs de guerre 1746 Et 1747. 》f 95-116, pp. 170-228.

Voir aussi : 1747. 115-2. Québec, 20 octobre 1747. « Dépenses... L'accadie... 》f 368, p. 416.

Voir aussi : 1748. 118. Québec, 20 octobre 1748. «Memoire... $1746-1747 \ldots$... 18 , p. 27.

Voir aussi : 1747. 116-1. Québec, 23 octobre 1748. «Etat des sommes... 》f 144-150, pp. 258-268.

Voir aussi : 1745. 115-2. Québec, 30 octobre 1748. « Memoire... $1745 \ldots \gg$ f 251 , p. 211.

Voir aussi : 1744. 116-1. Québec, 30 octobre 1748. « Memoire... MVIIc quarante quatre... 》f 83-86, pp. 129-136.

Voir aussi : 1748. 1161. Québec, 1 novembre 1748. «Memoire... »f 71-72, pp. 113-115.

Voir aussi : 1748. 116-1. Québec, 1 novembre 1748. «... cons. truction de chantier... »f 77, p. 122. 
HISTOIRE ECONOMIQUE DU CANADA FRANÇAIS

Voir aussi : 1748. 116-1. Québec, 1 novembre 1748. «Estat... Nouveau Chantier... »f 78, pp. 123-124.

Voir aussi : 1748. 116-1. Québec, 1 novembre 1748. «...par Suplement... 》f 81, p. 127.

Voir aussi : 1748. 116-1. Québec, 1 novembre 1748. "Bordereau... acquits... 》f 122, 125, 132, pp. 215, 221, 238.

Voir aussi : 1748. 115-2. Québec, 3 novembre 1748. «Memoire... 》f 365 , p. 411.

Voir aussi : 1748. 118. Détroit, 12 juin 1749. «Memoire...» f 378 , p. 146.

Voir aussi : 1749. 116-2. Québec, 27 octobre 1750. «Balance des fonds... »f 332-335, pp. 201-205.

\author{
Cameron NISH, \\ professeur à l'Université Sir George Williams \\ et \\ directeur de recherche, \\ Centre de Recherches en Histoire économique \\ du Canada français.
}

